\title{
Characterization of Layered Structures of Ti-6Al-4V Alloy and Metal Matrix Composites on Its Base
}

\author{
S.V. Prikhodko ${ }^{\text {, P.E. Markovsky }}{ }^{2}$, D.G. Savvakin², O.O. Stasiuk ${ }^{2}$, M. Norouzi Rad ${ }^{3}$, C. Choi ${ }^{1}$, O.M. \\ Ivasishin $^{2}$ \\ 1. Department of Materials Science and Engineering, UCLA, Los Angeles, CA, USA \\ 2. G.V. Kurdyumov Institute for Metal Physics, National Academy of Science of Ukraine, Kiev, Ukraine \\ 3. Carl Zeiss Microscopy LLC., Pleasanton, CA, USA
}

High specific strength of Ti-based alloys makes them highly demanded materials for various structural applications [1]. Alloys reinforcement with light $\mathrm{TiC}$ and $\mathrm{TiB}$ creates a metal matrix composite (MMC) with higher hardness without compromising the low weight of the material, however inevitably jeopardizing their plasticity. The layered structures which are used the MMC at the surface and the alloy (without reinforcement) at the core is an attractive way to fabricate the materials with improved external hardness deprived of yielding their internal ductility.

Two- and three-layers structures were fabricated in this study on the base of Ti-6Al-4V (wt.\%) (Ti-64) alloy and its MMC reinforced with fine $\mathrm{TiB}$ and $\mathrm{TiC}$ particles. Samples were prepared using blended elemental powder metallurgy $(\mathrm{BEPM})$. Samples with the shape of plates $(90 \times 90 \times 18)$ bars $(65 \times 10 \times 10)$ and cylinders $(\varnothing 10 \times 12)$ (all in $\mathrm{mm})$ were die-pressed at 150, 400 and $650 \mathrm{MPa}$ correspondingly and sintered in vacuum $\left(1250{ }^{\circ} \mathrm{C}, 4 \mathrm{~h}\right)$. After the sintering samples were subjected to a hot pressing and annealing. More details on samples fabrication can be found elsewhere [2]. Structure of the samples was characterized using light optical microscopy, SEM Nova 230 (ThermoFisher) equipped with EDS Noran 7 (ThermoFisher) and SEM Vega3 (Tescan). EBSD-EDS study was performed on the AZtec (Oxford Instruments) system coupled with the SEM LEO 1550VP (Zeiss) operated at $20 \mathrm{kV}$. X-ray microscopy was conducted on submicron resolution XRM, Versa 520 (Zeiss). TEM was performed on 100CX (JEOL) using accelerating voltage $100 \mathrm{kV}$. Samples for the SEM, EBSD were prepared by mechanical polishing using MultiPrep system (Alligh). TEM samples were prepared by punching $3 \mathrm{~mm}$ disks followed by their thinning and polishing using MultiPrep and their final perforation using the ion beam system PIPS (Gatan).

Results show that relatively big size multi layered structures on the base of Ti-64 alloy and its MMC with $\mathrm{TiC}$ and $\mathrm{TiB}$ reinforcement particles can be successfully fabricated using BEPM. It was found that used powder size and the hydrogen content in initial Ti powders (hydrogenated titanium or conventional titanium powders) are critical parameters to prevent the layered structures distortion and delamination during their sintering. Multi-layered materials were characterized with uniform structure, chemical composition and full integration between the layers. Post-sintering hot pressing was effective to reduce porosity level in the layered structures, which used MMC with TiC. That improved the mechanical properties. The effect of hot pressing and annealing on the properties of the layered plates with TiB MMCs was less apparent due to distinctively different structure of MMCs with TiB and TiC, which was revealed by $3 \mathrm{D}$ imaging using $\mathrm{X}$-ray microscopy.

\section{References:}

[1] G. Lutjering, and J.C. Williams, 2003, Titanium

[2] S.V. Prikhodko et al, Microsc. Microanal. 23 (S1) (2017), p. 322. 


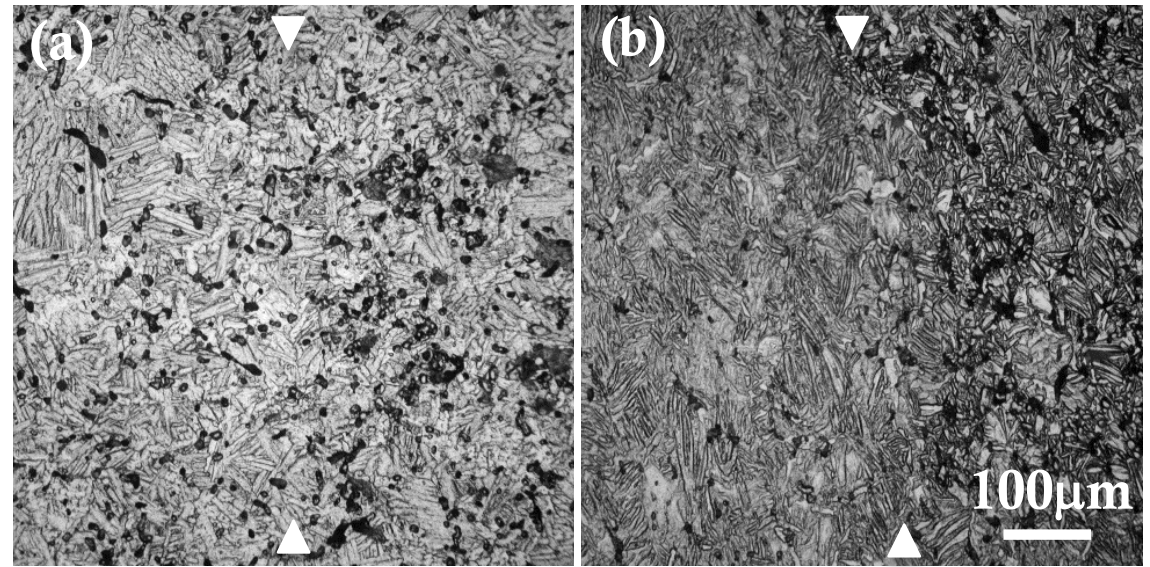

Figure 1. Light optical microscopy images of bi-layered structures based on Ti-64 alloy fabricated using die-pressing at $150 \mathrm{Mpa}$ followed by the sintering $\left(1250{ }^{\circ} \mathrm{C}, 4 \mathrm{hrs}\right)$. Images show the structure close to the interface (marked) between the layer of Ti-64 alloy (left) and MMC (right) on its base. The MMC has $5 \%$ of $\mathrm{TiC}$. Image (a) is as-sintered structure and (b) is the structure after additional hot plastic deformation on $\sim 37 \%$. Both images are shown at the same magnification.
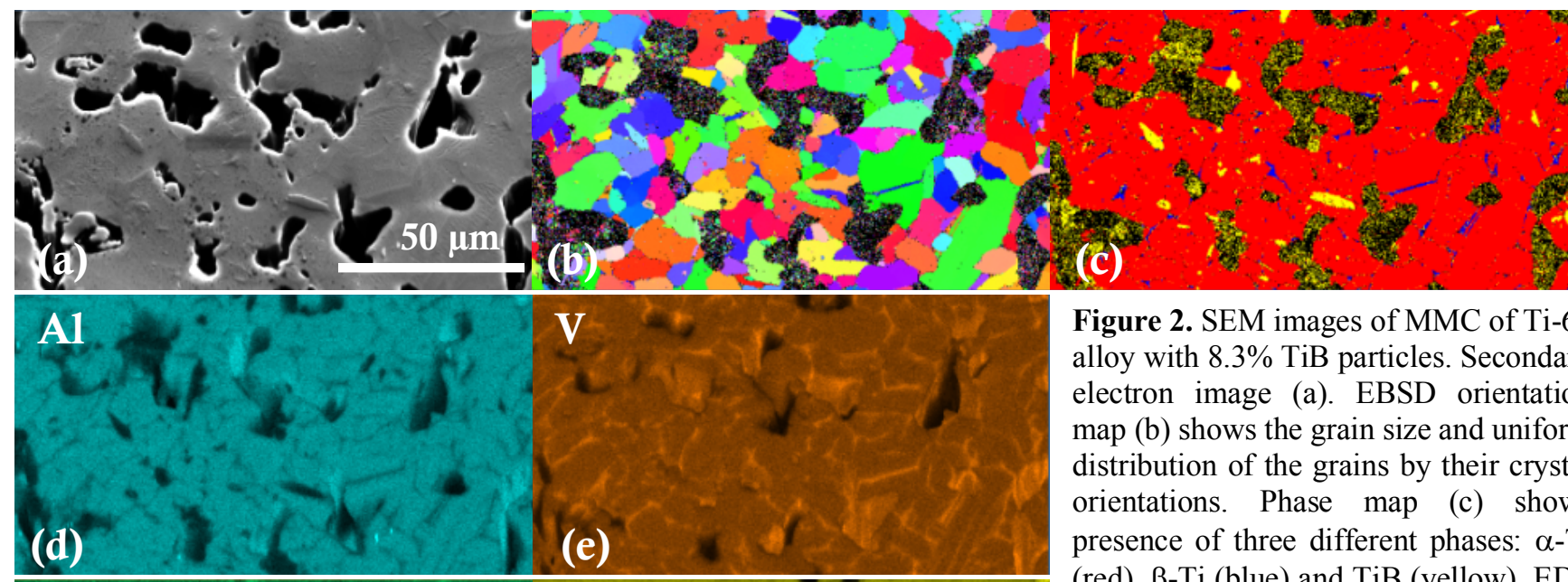

Figure 2. SEM images of MMC of Ti-64 alloy with $8.3 \%$ TiB particles. Secondary electron image (a). EBSD orientation map (b) shows the grain size and uniform distribution of the grains by their crystal orientations. Phase map (c) shows presence of three different phases: $\alpha$-Ti (red), $\beta$-Ti (blue) and TiB (yellow). EDS

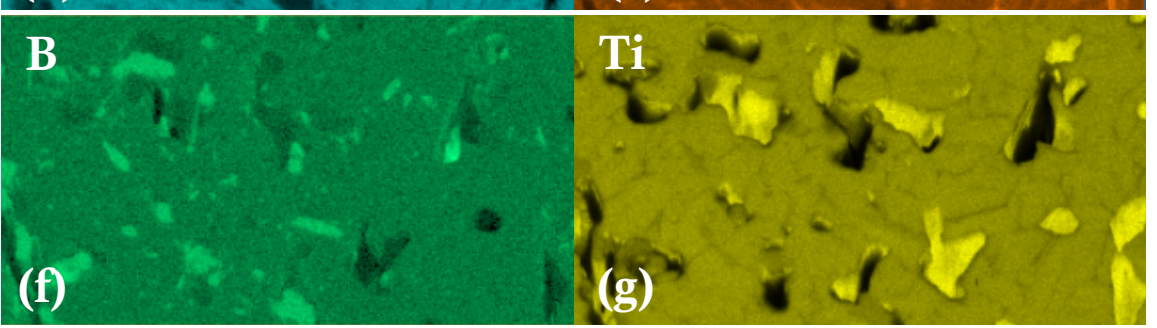
Elemental maps (d-g) shows distribution of $\mathrm{Al}, \mathrm{V}, \mathrm{B}$ within $\mathrm{Ti}$ matrix (some topographical effect due to porosity is present on those images).
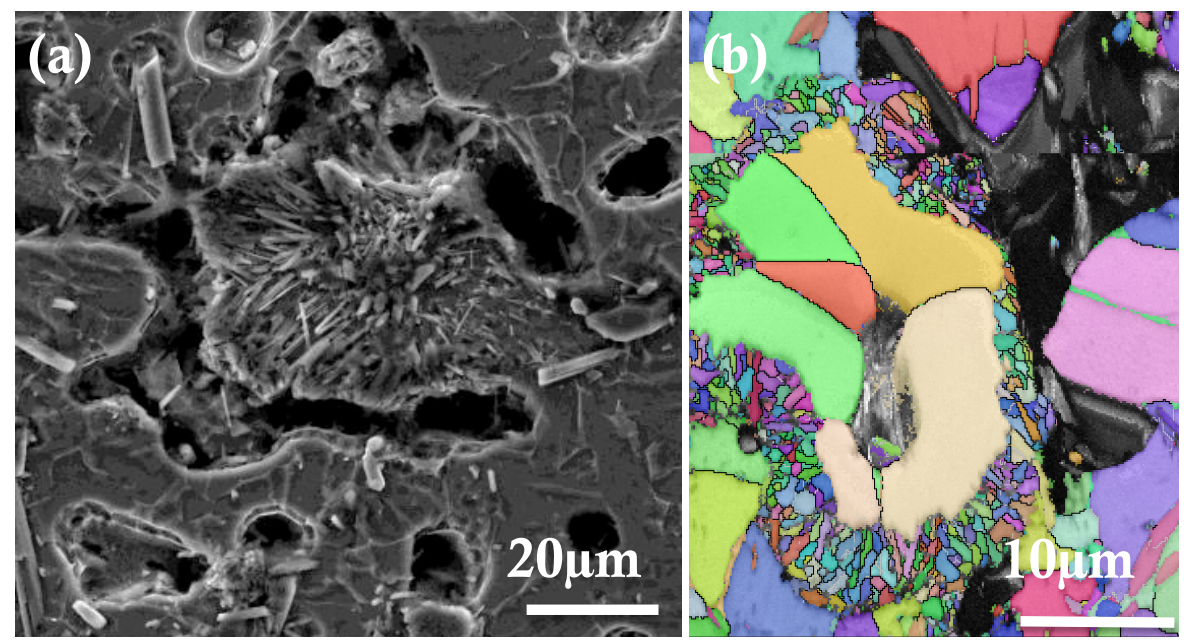

Figure 3. Secondary electron SEM image (a) and EBSD orientation map (b) of titanium boride inclusion. "Sea urchin" morphology of Ti borides indicative of $\mathrm{TiB}_{2}$ presence. High resolution EBSD map shows that the inner area of inclusion still has some not transformed $\mathrm{TiB}_{2}$ crystals, which are covered by the layer of $\mathrm{TiB}$ needles (the chain of fine crystals in (b)). "Sea urchin" morphology particles are surrounded by extensive pore formations. X-Ray microscopy results are in good compliance with those findings [2] 